\title{
Preservation of Triceratops horridus Tissue Cells from the Hell Creek Formation, MT
}

\author{
Mark H. Armitage
}

Electron Microscopy Laboratory, Micro Specialist, 587e North Ventu Park Road, STE 304, Thousand Oaks, CA 91320

micromark@juno.com

\begin{abstract}
Dinosaur soft tissues are shown to be remarkably preserved to the sub-micron level of ultrastructure despite environmental and biological factors associated with burial for millions of years. Light microscopy and scanning electron microscopy (SEM) reveals soft tissue features such as fibrillar bone tissue, osteocytes, and blood vessels. Concerns that these findings relate to contamination or biofilm formation have been refuted. Notwithstanding the controversial nature of these discoveries, soft dinosaur tissues should be systematically searched for and thoroughly characterized in other dinosaur remains.
\end{abstract}

\section{Introduction}

Remarkably preserved cells and tissues from dinosaurs have been reported since the mid 1960s [1], however until recently, dinosaur bone specimens usually have not been decalcified or otherwise destructively studied for the presence of soft tissues because complete bone specimens are highly prized by paleontologists and collectors. Over the past 50 years, soft blood vessels, collagen bands, intact cells, bone cells (osteocytes), filopodia with primary and secondary branching, cell-to-cell junctions, intracellular nuclei, and other soft tissue details have been observed and illustrated from various different species of dinosaurs including Tarbosaurus bataar, Tyrannosaurus rex, Brachylophosaurus canadensis, and Triceratops horridus. [1-6]. Initial criticisms, which labeled these soft structures as biofilms [6], have been resolved as incorrect [7].

In 2012 I collected a large Triceratops horridus supraorbital horn from the Hell Creek Formation at Glendive, Montana. The horn yielded soft sheets of fibrillar bone (Figure 1) and life-like cells. A Triceratops rib specimen from the same deposit contained soft blood vessels and red blood cell-like (RBC) microstructures. Remarkable preservation of individual bone osteocytes encapsulated within the stretchy sheets of fibrillar horn bone was observed, as were osteocytes positioned upon sheets of fibrillar bone adhering to permineralized vessels within the decalcified horn bone [6]. Variable-pressure scanning electron microscopy (VPSEM) of uncoated specimens was not attempted at that time, nor were individual cells isolated from the specimen for further analysis. In this article I describe VPSEM and cell isolation results from the Triceratops horn.

\section{Materials and Methods}

Specimen preparation. The hand-sized pieces of the horn, somewhat "pie-slice" in shape and extending from the exterior horn surface to the inner trabecular (cancellous) bone (core), were fixed in a $2.5 \%$ solution of glutaraldehyde, buffered with $0.1 \mathrm{M}$ sodium cacodylate buffer at $4^{\circ} \mathrm{C}$ for 5 days, rinsed in distilled water and buffer, and stored in phosphate buffered saline (PBS). Pieces, roughly $20 \mathrm{~mm}^{3}$ in size, were extracted from the inner bone core by pressure fracture and were processed through a decalcification protocol as follows: bone pieces were rinsed in pure water after fixation and were incubated in a solution of $14 \%$ sodium ethylenediamine tetraacetic acid (EDTA) at room temperature. The EDTA was exchanged every 2 to 4 days for a period of 4 weeks after which bone fragments were processed for scanning electron microscopy (SEM).

Other pieces were soaked for 4 months in EDTA. Even after this treatment significant bone mineral/hardened material remained; therefore, it is unknown whether complete decalcification in EDTA would yield soft and transparent, vessel-like tissues, such as previously reported [7-11]. A soak in hydrofluoric acid (HF) was not attempted, but it might prove more successful in liberating any soft vessels that remain. Rib specimens were similarly fixed, washed, and pressure fractured to reveal inner surfaces of compact bone (Figures 2 and 3).

Light microscopy of cells. Aliquots of decalcification solutions (post soak) were transferred by pipette into tied off chambers of Snakeskin dialysis tubing, (Thermo Scientific, Rockford, IL) and were submerged into vials of distilled water for 2 weeks. Water was exchanged every 2 days, and after 2 weeks cells were transferred after dialysis onto glass microscope slides for examination and imaging on a Jenaval light microscope (Carl Zeiss Jena) equipped with a Jenoptik ProgRes (Jena, Germany) C14plus camera.

SEM imaging of bone. After a 4-week soak in EDTA, decalcified bone was air-dried and affixed to aluminum stubs. For Figures 2-4, bone specimens were sputter-coated with

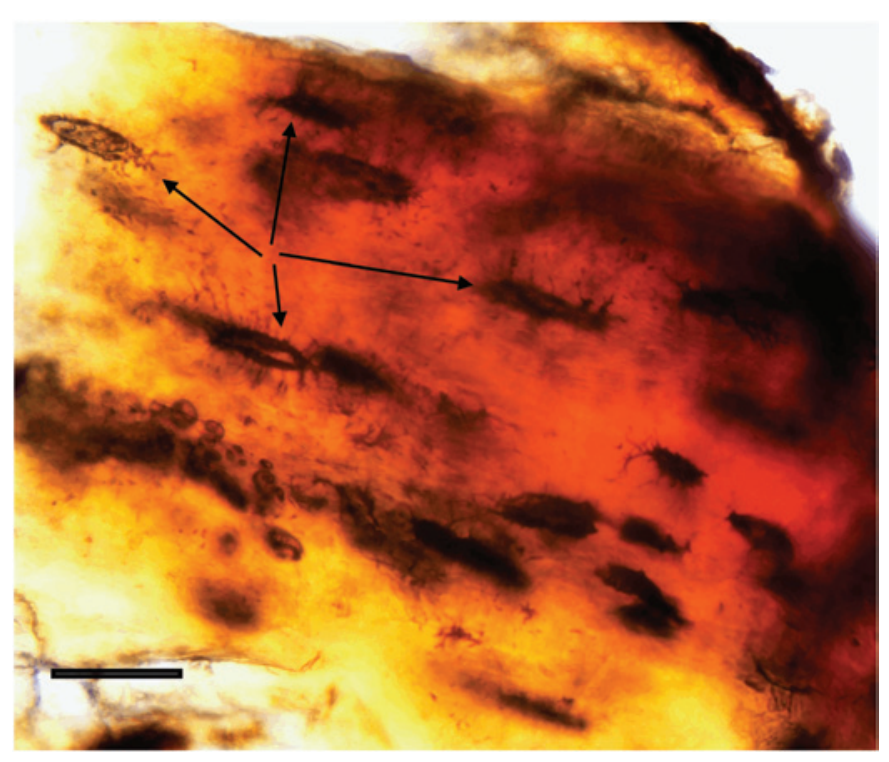

Figure 1: Portion of soft, stretchy fibrillar bone from Triceratops horn. Note embedded osteocytes (black arrows). Scale bar $=30 \mu \mathrm{m}$. 


\section{SEEING the BIG PICTURE}

How did your role as Sustainable Laboratories Coordinator lead to an interest in the field of microscopy?

I work with the sustainability aspects of scientific research, particularly the facility costs (water, electricity, chemicals, etc.). Microscopes are essential research tools and so most facilities I work with own and use them. I was curious as to what opportunities for efficiencies or cost savings there might be.

What are the challenges in driving adoption of modern efficient lighting technologies?

The overall challenge is always convincing users that the hardware can deliver for a price that makes sense.

"Illumination for research-grade microscopy presents the particular challenge of having a wide range of technical requirements (wavelength, power, spatial and temporal distribution)."

From my side, I first wanted to know that users were happy with the technical features of Lumencor light engines. I can't promote something they don't actually want. After organizing the purchase of 5 Lumencor light engines for the University of Edinburgh I'm happy to report l've heard nothing but praise for the new systems.

\section{www.lumencor.com}

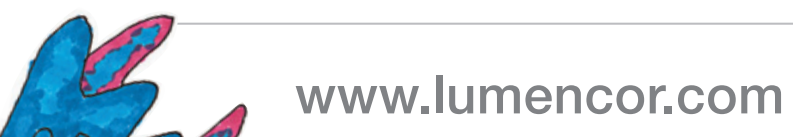

How have Lumencor's products addressed these challenges?

From the feedback of researchers, Lumencor manufactures some of the most powerful solid-state illumination systems available. These systems may entail a significant purchase-cost increase when compared to mercury-arc systems. But in the big picture, whole-life costing of the systems produces a different conclusion.

"When bulb costs and unproductive idle time are factored in, purchasing a solid-state light source can save $£ 8,000-£ 25,000(\$ 12,000-\$ 40,000)$ over its projected working life compared to mercury-arc sources."

This doesn't factor in mechanical cooling savings from reduced heat output of Lumencor light engines, which would increase savings. Importantly the bulk of these savings go back to the researcher, as they pay for bulb costs. At the University of Edinburgh, we purchased 5 Lumencor light engines instead of mercury-arc lamps, saving researchers a minimum of $£ 40,000(\$ 60,000)$ over the equipment lifetime, while at the same time eliminating the repetitive tasks of mercury bulb replacement and disposal.

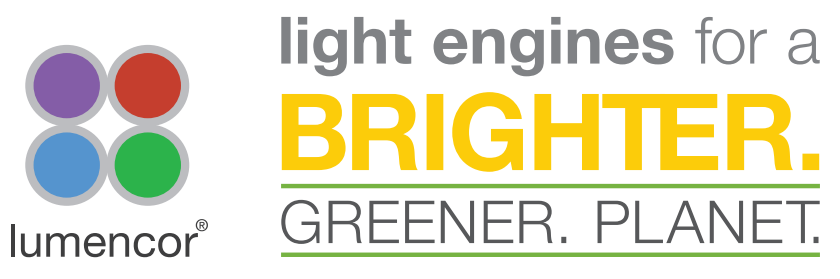

\section{light engines for a BRIGHTER. GREENER. PLANET.}
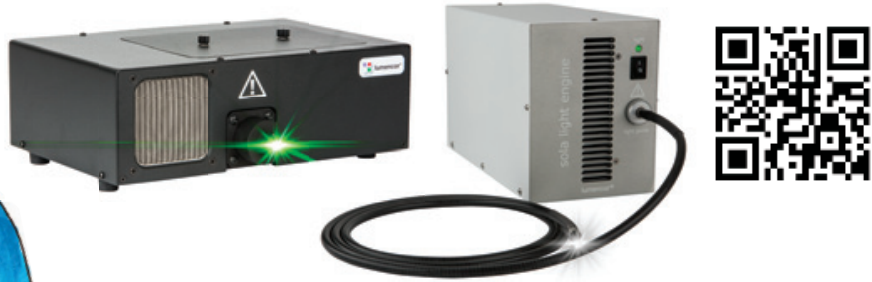


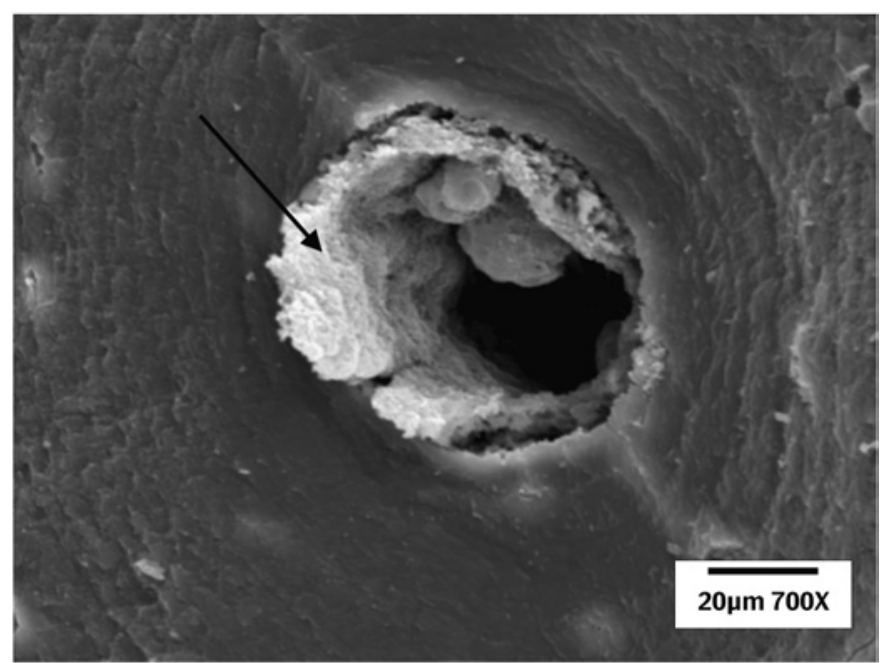

Figure 2: Soft blood vessel wall extending from Haversian canal (black arrow), Triceratops rib. Scale bar $=20 \mu \mathrm{m}$.

gold for 90 seconds at $20 \mathrm{~mA}$ and were imaged at $30 \mathrm{kV}$ under high vacuum in a Hitachi S2500 SEM. Bone specimens in Figures 5-6 were left uncoated and imaged at $10 \mathrm{kV}$ in a Zeiss EVO SEM with a backscatter electron detector. Bones in Figures 7 and 8 were left uncoated by metal and imaged in a Zeiss EVO SEM at $3.1 \times 10^{-4} \mathrm{~Pa}$ with a secondary electron detector.

\section{Results}

Fibrillar Bone. Soft, stretchy sheets of fibrillar bone tissue exhibited layers of bone cells (osteocytes). These would come into focus depending on the layer imaged in light microscopy (Figure 1).

Blood vessels. Soft blood vessels (arrow in Figure 2) extended from many Haversian canals in the fractured rib. It is clear that when the living vessel was fully extended by blood and serum, it tightly abutted against the undulations in the Haversian canal wall. Spherical microstructures, consistent with the size and shape of RBCs, were observed in the lumens of many vessels in non-decalcified Triceratops rib specimens

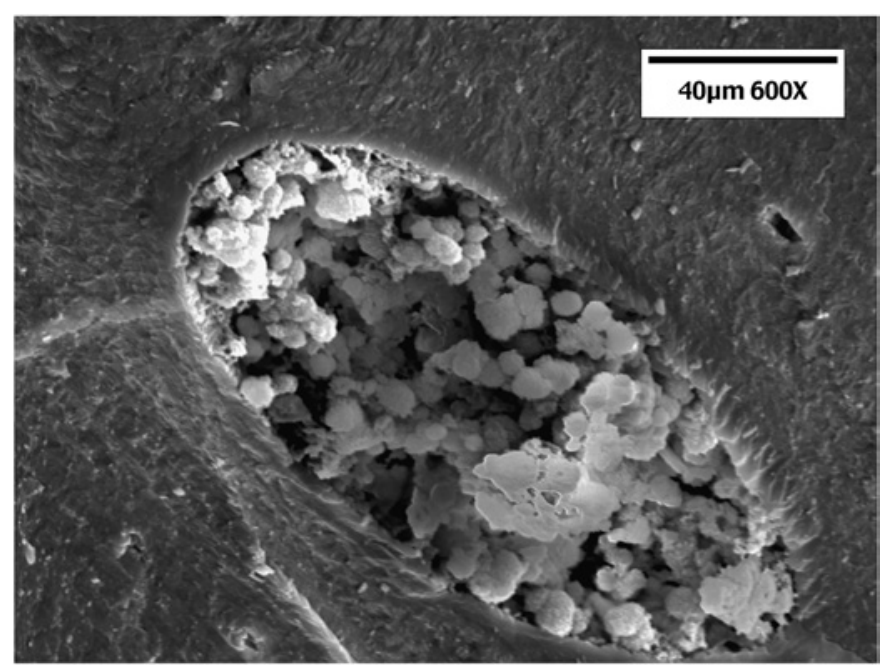

Figure 3: Spherical microstructures, consistent with size of RBCs, within Haversian canal of Triceratops rib. Scale bar $=40 \mu \mathrm{m}$.

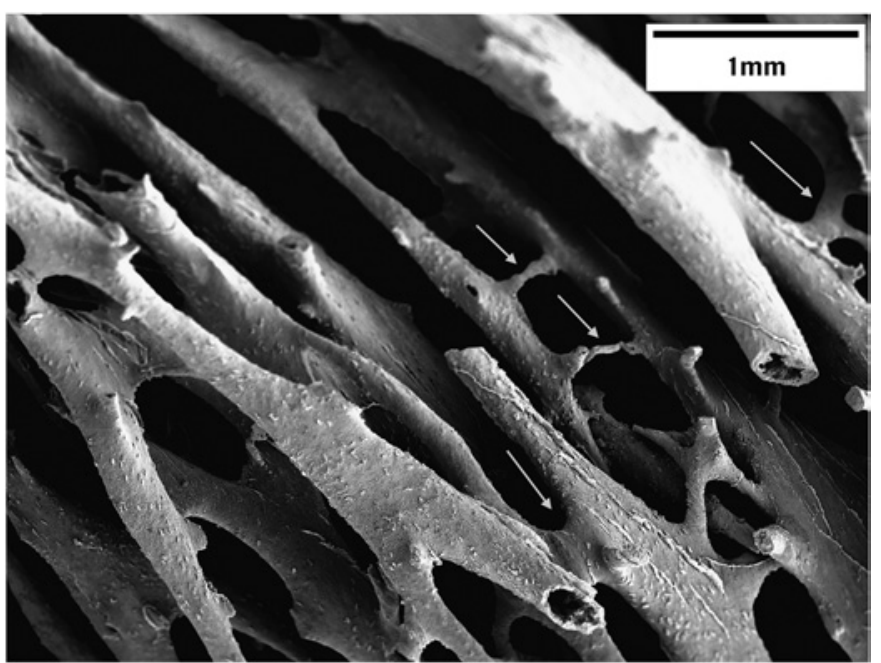

Figure 4: Blood vessels from decalcified sample of Triceratops horn. Cross-linking Volkman's canals are evident (white arrows). It was on the surfaces of these blood vessels where soft bone osteocytes were found (discrete dots on vessels). Scale bar $=1 \mathrm{~mm}$.

(Figure 3). The amount of rib material collected did not allow for decalcification experiments, therefore it is unknown if large regions of soft vessels within the rib were preserved.

Horn blood vessels appeared to be permineralized after decalcification removed the bone mineral (Figure 4). Volkmann canals were present (Figure 4 arrows), and the outside surfaces of vessels were wrapped in soft fibrillar bone sheets. Figures 4 and 5 show many osteocytes on the surface of these sheets as well as within adjoining sheets of fibrillar bone (small white dots on the surfaces of vessels, in Figure 4 and arrows on Figure 5).

Bone cells. Osteocytes imaged with SEM exhibited smoothly tapered filopodia, which extended to $20 \mu \mathrm{m}$ in length in some instances (white arrows in Figures 6 and 7). A band of preserved collagen is labeled with a black arrow in Figure 7.

\section{Discussion}

The remarkable preservation of delicate ultrastructures such as filopodia and cell-to-cell junctions (white arrows, Figures 6 and 7) has resisted a simple explanation despite hypothesized

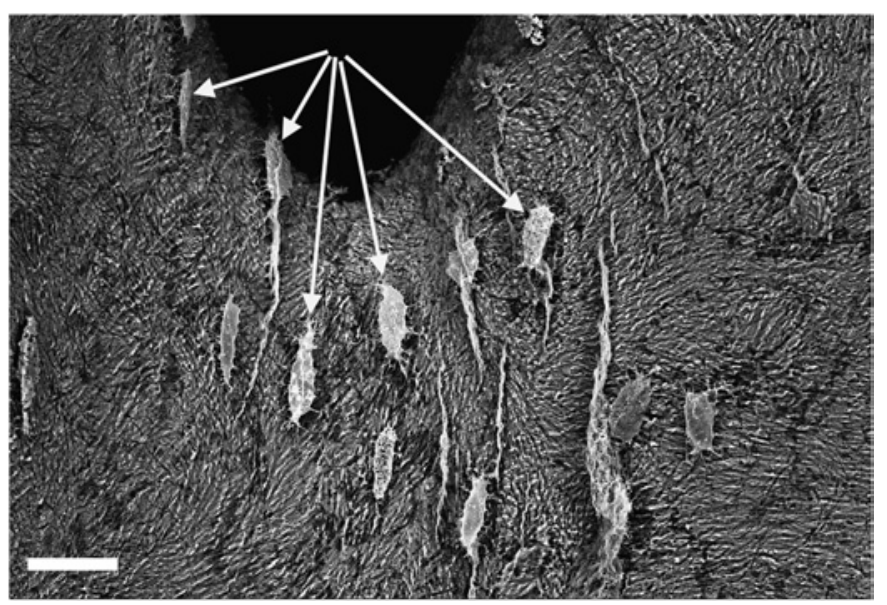

Figure 5: Numerous soft bone osteocytes are seen on the surface of a blood vessel (white arrows). Scale bar $=35 \mu \mathrm{m}$. 


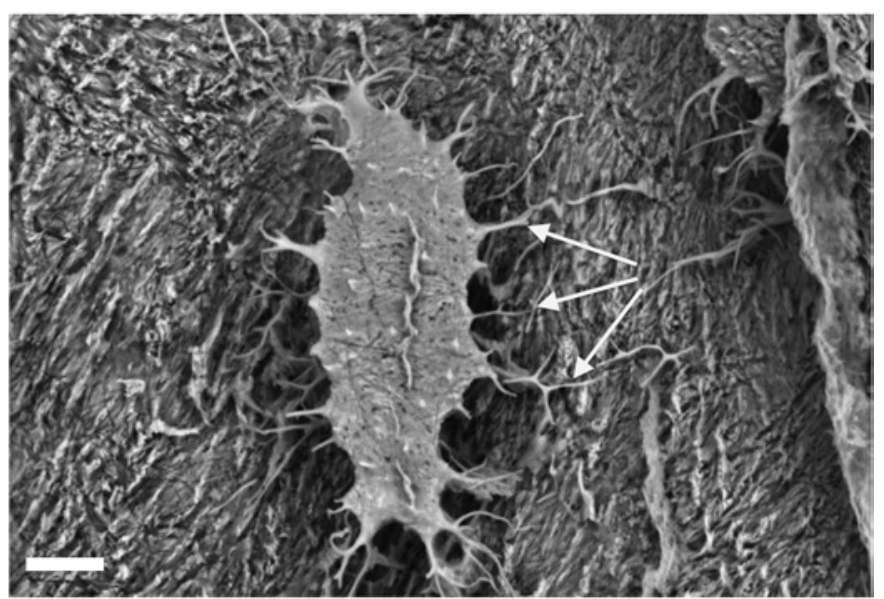

Figure 6: A single soft osteocyte from Triceratops horn. Delicate filipodia extend out to connect with other cells (white arrows). Scale bar $=4 \mu \mathrm{m}$.

temporal limits on molecular preservation over millions of years [13]. In the case of soft vessels recovered from dinosaur femur specimens, it seems reasonable that these tissues were sequestered from the elements and from biological scavenging activity because of deep encapsulation within compact bone. Within the Triceratops horn, however, which was highly vascular, no sequestration was likely because all of the vessels were openly exposed to air, soil, water, scavengers, dissolved salts and minerals, and the freeze-thaw cycle and heat of Montana seasonal weather; yet a high degree of preservation persists. While plant roots, fungal hyphae, and insect remains were all found traversing the horn, soft fibrillar sheets of bone and well-preserved osteocytes remain.

Discoveries of soft blood vessels, RBC-like microstructures, and soft bone osteocytes have been controversial [8-10]. One criticism maintained that these soft tissue discoveries are not endogenous tissues but rather the remains of bacterial biofilms, which "retain much of the original morphology" of dinosaur bone osteocytes and filipodia [8]. However osteocytes from other dinosaur specimens were later demonstrated to contain actin, tubulin, and histone $\mathrm{H} 4$ proteins, which are not found in bacteria or bacterial biofilms. These osteocyte proteins are consistent with other dinosaur protein finds [7].

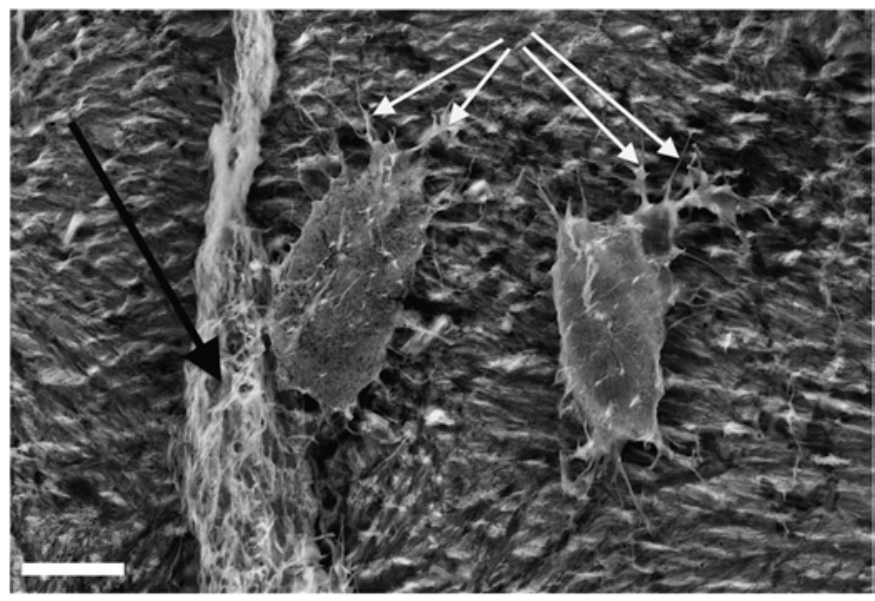

Figure 7: Fine structure filipodia extend as dendrites from two soft osteocytes in Triceratops horn (white arrows). Black arrow is pointing to a band of collagen. Scale bar $=10 \mu \mathrm{m}$.

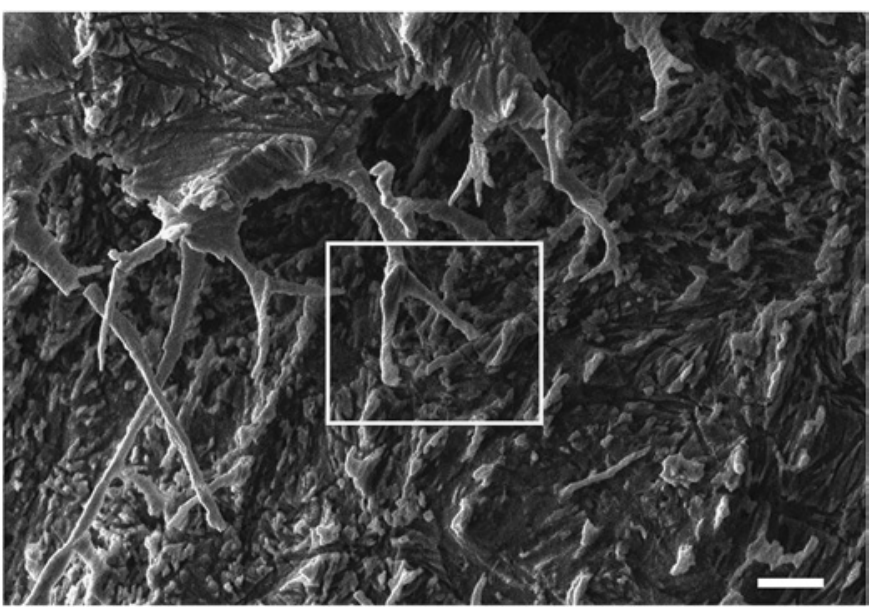

Figure 8: White box surrounding delicate bifurcated termination of filipodia. Smooth edges of dendrite indicates the high degree of preservation. Scale bar $=7 \mu \mathrm{m}$.

Uncoated specimens of decalcified bone in this study yielded osteocytes with a higher degree of ultrastructural preservation than previously reported $[3,6,9,12]$. Uncoated bone surfaces show lacunae depressions (Figures 5-7), extensive filopodia (Figures 6-10), collagen aggregates (Figure 7), and cell surfaces displaying the indented impressions of overlying and compressing bone (Figures 6 and 8).

Figures 9 and 10 show cells that were successfully isolated from fibrillar bone. In future work, it is hoped that individual cells such as these can be examined using immunohistochemistry for the presence of endogenous proteins.

\section{Conclusion}

Claims of contamination and biofilm replication have been dismissed $[7,12]$, and identification of intra-cellular and intra-nuclear proteins have been verified showing that these are endogenous dinosaur tissues [12]. Therefore claims that these are not original dinosaur tissues appear to be questionable.

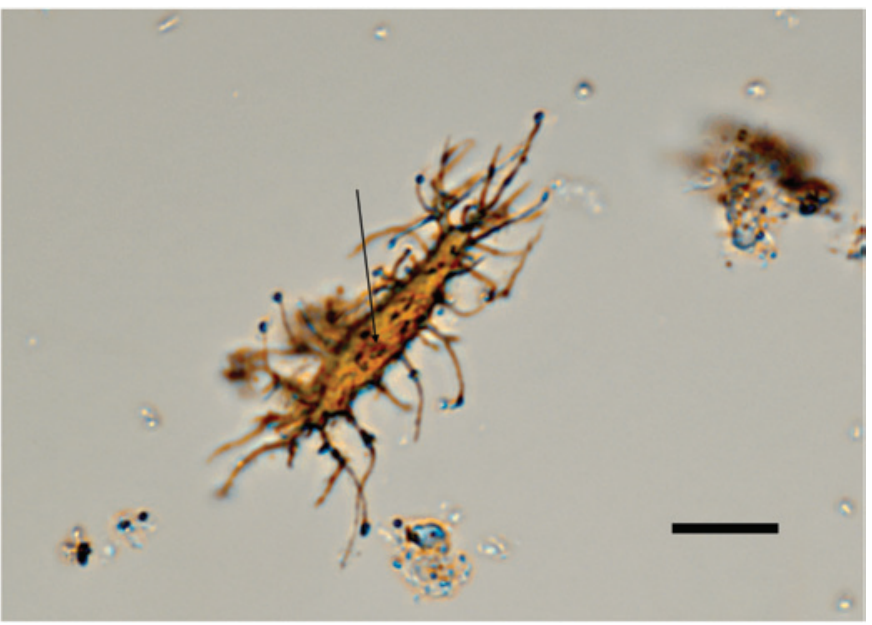

Figure 9: Isolated and washed Triceratops soft bone osteocyte under light microscopy. Cells often contained nucleus-like microstructures (black arrow). Scale bar $=5 \mu \mathrm{m}$. 


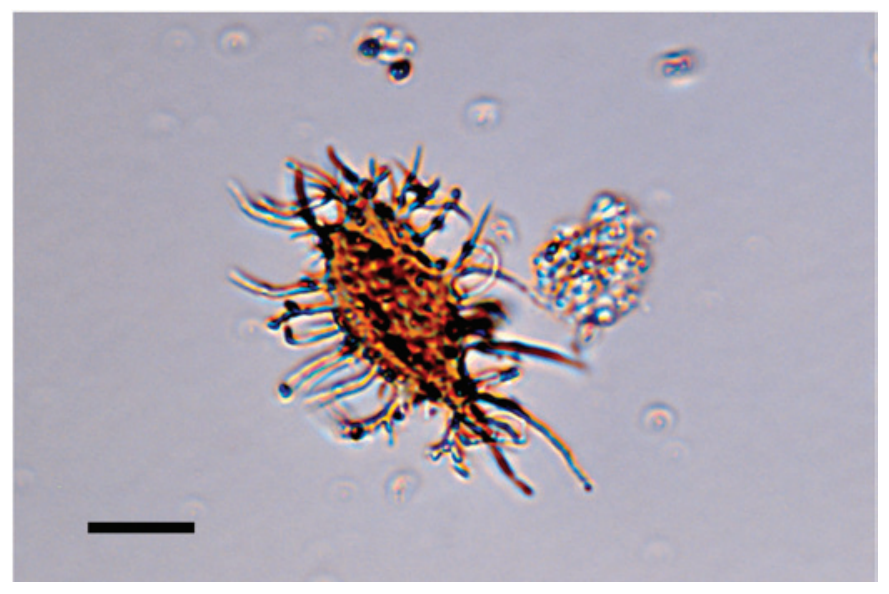

Figure 10: Isolated and washed Triceratops soft bone osteocyte under light microscopy. Note many elongated filipodia. Scale bar $=8 \mu \mathrm{m}$.

Original dinosaur soft tissues are shown here to be remarkably preserved to the sub-micron level of ultrastructure despite the environmental and biological factors associated with its burial. Notwithstanding the controversial nature of these discoveries, soft dinosaur tissues should be systematically searched for and thoroughly characterized in other dinosaur remains.

\section{Acknowledgements}

I thank the following: Carl Zeiss SMT personnel for assistance in acquiring and collecting images of uncoated
Triceratops bone osteocytes using the EVO environmental SEM; Norm Burns, senior application specialist (consultant); Ken Robinson, senior application specialist; and KD Derr, electron and ion microscopy specialist. I am indebted to Philip Oshel and Charles Lyman for critical comments, which made this a much better article. I also thank Per Larson and Brent Fjarli for funding.

\section{References}

[1] R Pawlicki et al., Nature 211(5049) (1966) 655-57.

[2] R Pawlicki, Acta Anat 100 (1978) 411-18.

[3] R Pawlicki and M Nowogrodzka-Zagorska, Ann Anat 180 (1998) 73-77.

[4] MH Schweitzer and J Horner, Ann Paleon 85 (1999) 179-92.

[5] L Zylberberg and M Laurin, C R Paleovol 10 (2011) 357-66.

[6] MH Armitage and KL Anderson, Act Histo 115 (2013) 603-08.

[7] MH Schweitzer et al., Bone 52 (2013) 414-23.

[8] TG Kaye et al., PLoS One 3(7) (e2008).

[9] MH Schweitzer et al., Science 307 (2005) 1952-55.

[10] MH Schweitzer, JL Wittmeyer, and JR Horner, Proc R Soc 274 (2007) 183-97.

[11] MH Schweitzer et al., Science 316 (2007) 277-80.

[12] MH Schweitzer et al., Science 324 (2009) 626-31.

[13] MH Schweitzer et al., Proc Royal Sci B 281 (2014) 3-10.

The Department of Materials Science and Engineering at The Ohio State University (OSU) invites applications for a tenure-track faculty position in advanced imaging and analysis of materials using electron microscopy, ion beam microscopy and/or atom probe tomography.

We welcome outstanding applicants with interests in areas such as multiscale correlative imaging, high-resolution imaging, analytical electron microscopy, advanced tomography and in-situ experimentation for the characterization of materials. Appointment will be at assistant or associate professor level depending on the qualifications and experience of the selected candidate.

This position is associated with the Center for Electron Microscopy and Analysis (CEMAS, cemas. osu.edu), a multi-million dollar, world-leading facility to drive innovative teaching, learning, and research in materials for engineering, medicine, and physical and biological sciences. This position is partially funded by the OSU Discovery Themes, a significant faculty hiring investment in key thematic areas in which the university can build on its culture of academic collaboration to make a global impact.

We seek a person who is an ardent discoverer and passionate teacher and mentor, with a demonstrated record of leadership and collaboration in an academic and/or R\&D environment. Candidates must have an established record of accomplishment in electron microscopy research and a doctoral degree in materials science and engineering or a related field.

The successful candidate will be expected to develop and sustain active sponsored research programs, teach core undergraduate and/or graduate courses, and develop new graduate courses related to their research expertise. The candidate should have experience developing or working in interdisciplinary research teams and experience mentoring members of underrepresented groups. The anticipated start date is August 2016. Screening of applicants will begin immediately and priority consideration will be given to applications submitted by January 1,2016 .

Interested candidates should submit a complete curriculum vitae, separate 2-3 page statements of research and teaching goals, and the names, postal addresses, and e-mail addresses of four references electronically to: eng-mse-facultysearch4@osu.edu

Ohio State is an equal opportunity employer and is committed to establishing a culturally and intellectually diverse environment, encouraging all members of our learning community to reach their full potential. All qualified applicants will receive consideration for employment without regard to race, color, religion, sex, sexual orientation or identity, national origin, disability status or protected veteran status. We are responsive to dual-career families and strongly promote work-life balance to support our community members through a suite of institutionalized policies. We are an NSF Advance Institution and a member of the Ohio/Western Pennsy/vania/West Virginia Higher Education Recruitment Consortium. 


\section{STELடAR 月EதபLTS}

\section{$\Delta T$

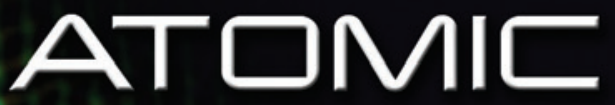 \\ SCALE}

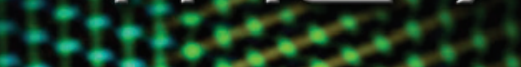

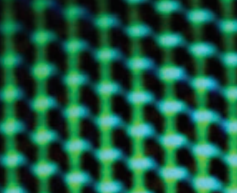

for

\section{ARM-200F • ARM-300F Atomic Resolution TEM}

- Highest resolution

commercially-available S/TEM

- 200kV and 300kV models

- $\mathrm{C}_{\mathrm{s}}$ correctors - innovative options

- Cold FEG

- Superior SDD technology

- Ultimate stability
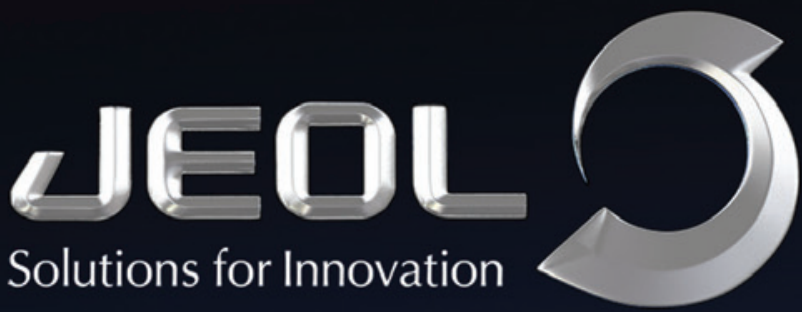

Solutions for Innovation

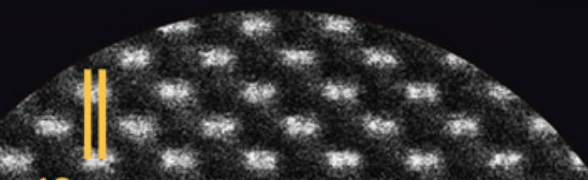

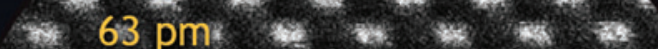

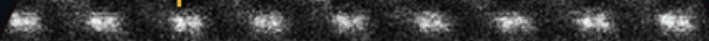

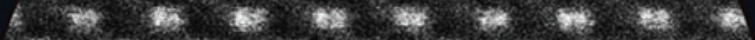

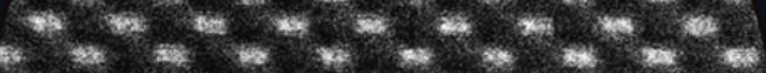

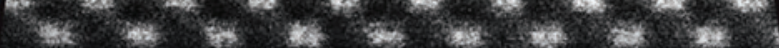

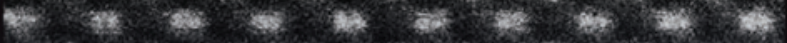

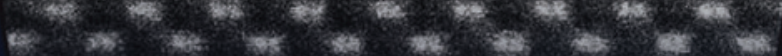

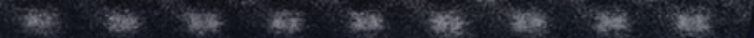

\section{Learn more at jeolusa.com/atomicresolutionTEM}

A leader never stops innovating

www.jeolusa.com • salesinfo@jeol.com 